\title{
STABILITY OF POLYNOMIAL CONVEXITY OF TOTALLY REAL SETS
}

\author{
FRANC FORSTNERI ${ }^{1}$
}

\begin{abstract}
We show that certain compact polynomially convex subsets of $\mathbb{C}^{n}$ remain polynomially convex under sufficiently small $\mathbf{C}^{2}$ perturbations.
\end{abstract}

1. Statement of the results. Let $M$ be a Stein manifold. Denote by $\mathcal{O}(M)$ the algebra of all holomorphic functions on $M$ with the standard topology of uniform convergence on compact subsets. A compact subset $K$ of $M$ is said to be $\mathcal{O}(M)$-convex if for every point $x \in M \backslash K$ there is a holomorphic function $f \in \mathcal{O}(M)$ such that

$$
|f(x)|>\sup _{y \in K}|f(y)|
$$

Since the holomorphic polynomials are dense in the algebra $\mathcal{O}\left(\mathbb{C}^{n}\right)$ of holomorphic functions on $\mathbb{C}^{n}$, an $\mathcal{O}\left(\mathbb{C}^{n}\right)$-convex subset of $\mathbb{C}^{n}$ is just a polynomially convex subset.

Given a compact $\mathcal{O}(M)$-convex subset $K$ of $M$, an open neighborhood $U$ of $K$ and a $\mathbf{C}^{k}$ diffeomorphism $\Psi$ of $U$ onto an open subset $\Psi(U)$ in $M$, we ask whether the set $\Psi(K)$ is also $\mathcal{O}(M)$-convex provided that $\Psi$ is sufficiently close to the identity on $U$ in the $C^{k}$ sense. In other words, is $\mathcal{O}(M)$-convexity a stable property under smooth perturbations? In general this is not so as the following example shows.

EXAmple 1. Let $M=\mathbb{C}^{2}$ and $K=\left\{(z, 0) \in \mathbb{C}^{2}:|z| \leqslant 1\right\}$. Clearly $K$ is convex and hence polynomially convex. The diffeomorphisms $\Psi_{\varepsilon}: \mathbb{C}^{2} \rightarrow \mathbb{C}^{2}$ given by

$$
\Psi_{\varepsilon}(z, w)=\left(z, w+\varepsilon|z|^{2}\right), \quad \varepsilon \geqslant 0,
$$

are close to the identity in the $\mathbf{C}^{\infty}$ sense for small $\varepsilon$, but the set

$$
\Psi_{\varepsilon}(K)=\left\{\left(z, \varepsilon|z|^{2}\right):|z| \leqslant 1\right\}
$$

is not polynomially convex for any $\varepsilon>0$ since it contains the boundary of the analytic disk $\Delta_{\delta, \theta}=\left\{\left(z, \varepsilon \delta^{2} e^{i \theta}\right):|z| \leqslant \delta\right\}$ for each $\delta \in[0,1]$ and $\theta \in \mathbb{R}$. These disks fill an open subset of $\mathbb{C}^{2}$ that is contained in the polynomial hull of $\Psi_{\varepsilon}(K)$ according to the maximum principle.

Received by the editors February 8, 1985 and, in revised form, April 22, 1985.

1980 Mathematics Subject Classification. Primary 32E20.

${ }^{1}$ Research supported in part by a Sloan Foundation Predoctoral Fellowship. 
Recall that a $\mathbf{C}^{1}$ submanifold $\Sigma$ of a complex manifold $M$ is called totally real if for each point $x \in \Sigma$ the tangent space $T_{x} \Sigma$ contains no nontrivial complex subspace. If $K$ is a compact subset of a totally real submanifold $\Sigma$, then by [2, p. 300] there is an open neighborhood $U$ of $K$ in $M$ and a $C^{2}$ strictly plurisubharmonic function $\rho: U \rightarrow \mathbb{R}_{+}$such that

$$
K=\{x \in U \mid \rho(x)=0\}, \quad \rho \geqslant 0 \text { strictly plurisubharmonic on } U \text {. }
$$

Conversely, every compact subset $K$ of $M$ of the form (1.1) is locally contained in a $\mathrm{C}^{1}$ totally real submanifold of $M$ [3]. Therefore we shall say that a compact subset $K$ of $M$ is totally real if it is of the form (1.1).

1.1 TheOREM. Let $M$ be a Stein manifold and $K$ a compact totally real subset of $M$ that is $\mathcal{O}(M)$-convex. Then every sufficiently small $\mathbf{C}^{2}$ perturbation of $K$ in $M$ is also $\mathcal{O}(M)$-convex.

We need to specify what we mean by a small $\mathbf{C}^{2}$ perturbation of $K$. We embed the Stein manifold $M$ in a Euclidean space $\mathbb{C}^{n}$ [4, p. 125]. Let $U$ be an open neighborhood of $K$ in $\mathbb{C}^{n}$, and denote by $E$ the Banach space $\mathbf{C}^{2}(U)^{n}$ of all $n$-tuples of complex valued functions $\Psi=\left(\Psi_{1}, \ldots, \Psi_{n}\right)$ of class $\mathbf{C}^{2}$ on $U$ which have finite norm

$$
\|\Psi\|_{E}=\sum_{j=1}^{n} \sup \left\{\left|D^{\alpha} \Psi_{j}(z)\right|: z \in U,|\alpha| \leqslant 2\right\} .
$$

Theorem 1.1 asserts that the set $\Psi(K)$ is $\mathcal{O}(M)$-convex for each $\Psi$ in an open neighborhood of the identity map in $E$ such that $\Psi(K) \subset M$.

1.2 Corollary. Let $M$ be a Stein manifold, let $N$ be a manifold of class $\mathbf{C}^{2}$ and let $B$ be an open neighborhood of 0 in some $\mathbb{R}^{m}$. Suppose that $F: N \times B \rightarrow M$ is a $\mathbf{C}^{2}$ map such that $F_{0}=F(\cdot, 0)$ is a totally real embedding of $N$ in $M$. If $K$ is a compact subset of $N$ such that $F_{0}(K)$ is $\mathcal{O}(M)$-convex, then $F_{t}(K)$ is $\mathcal{O}(M)$-convex for all $t$ in a neighborhood of 0 in $\mathbb{R}^{m}$. (Here, $F_{t}=F(\cdot, t)$.)

EXAMPLE 2. If $\Sigma$ is a totally real affine subspace of $\mathbb{C}^{n}$, then every compact subset $K$ of $\Sigma$ is polynomially convex. This follows from the Stone-Weierstrass approximation theorem and from the fact that the general linear group $\operatorname{GL}(n, \mathbb{C})$ acts transitively on the set of totally real subspaces of $\mathbb{C}^{n}$ of dimension $k$ for each $1 \leqslant k \leqslant n$. Hence, by Theorem 1.1, every small $\mathbf{C}^{2}$ perturbation of a compact subset $K \subset \Sigma$ is polynomially convex.

We shall consider the same question in the case when $K$ is a subset with nonempty interior in a Stein manifold. Suppose that $D$ is an open relatively compact subset of $M$ whose topological boundary $\bar{D} \backslash D$ contains a strictly pseudoconvex hypersurface $\Gamma$ such that $D$ lies on the convex side of $\Gamma$. More precisely, we assume 
that there is an open subset $V$ of $M$ and a strictly plurisubharmonic function $\rho$ : $V \rightarrow \mathbb{R}$ of class $\mathbf{C}^{2}$ such that

(i) $D \cap V=\{x \in V \mid \rho(x)<0\}$,

(ii) $\Gamma \cap V=\{x \in V \mid \rho(x)=0\} \subset \subset \Gamma$, and

(iii) $d \rho \neq 0$ on the set $\Gamma \cap V$.

We define the support of a diffeomorphism $\Psi: M \rightarrow M$ to be the closure of the set $\{x \in M \mid \Psi(x) \neq x\}$ where $\Psi$ differs from the identity map.

1.3 THEOREM. Let $D$ be an open relatively compact subset of a Stein manifold $M$ that satisfies the properties (i), (ii) and (iii) above. If the set $K=\bar{D}$ is $\mathcal{O}(M)$-convex, then for every sufficiently small $\mathbf{C}^{2}$ perturbation $\Psi: M \rightarrow M$ supported in $V$ the set $\Psi(K)$ is also $\mathcal{O}(M)$-convex.

1.4 COROLlaRY. If $D$ is a relatively compact strictly pseudoconvex domain in a Stein manifold $M$ such that $\bar{D}$ is $\mathcal{O}(M)$-convex, then every sufficiently small $\mathbf{C}^{2}$-perturbation of $\bar{D}$ in $M$ is also $\mathcal{O}(M)$-convex.

In $\S 2$ we prove Theorem 1.1 and Corollary 1.2 ; in $\S 3$ we prove Theorem 1.3 and Corollary 1.4 .

I wish to thank Professor Edgar Lee Stout for several helpful conversations.

\section{Polynomial convexity of totally real sets.}

Proof of TheOrem 1.1. If we embed the Stein manifold $M$ in a complex Euclidean space $\mathbb{C}^{n}$ [4], then a compact subset $K$ of $M \subset \mathbb{C}^{n}$ is $\mathcal{O}(M)$-convex if and only if it is polynomially convex. Therefore it suffices to prove the theorem in the case when $M=\mathbb{C}^{n}$. Let $U$ be an open subset of $\mathbb{C}^{n}, \rho$ a nonnegative strictly plurisubharmonic function on $U$, and let $K=\{z \in U \mid \rho(z)=0\}$ be a compact polynomially convex subset of $\mathbb{C}^{n}$. Choose a smooth function $\chi$ on $\mathbb{C}^{n}, 0 \leqslant \chi \leqslant 1$, such that $\chi=1$ on a neighborhood of $K$ and $\chi=0$ outside a compact subset of $U$. Let $E=\mathbf{C}^{2}(U)^{n}$ be the Banach space with the norm (1.2). Given a $\psi \in E$ we consider the map $\Psi: \mathbb{C}^{n} \rightarrow \mathbb{C}^{n}$ given by

$$
\Psi(z)=z+\chi(z) \psi(z) .
$$

Clearly $\Psi$ is proper. If the $E$-norm of $\psi$ is sufficiently small, then $\Psi$ is also regular and hence a covering projection. Since $\Psi$ is one-to-one outside a compact subset of $\mathbb{C}^{n}$, it has only one sheet and therefore it is a diffeomorphism of $\mathbb{C}^{n}$ onto $\mathbb{C}^{n}$. Every small perturbation of $K$ can be achieved within $U$ with a map of the form (2.1).

Choose a neighborhood $V$ of $K, \bar{V}$ contained in $U$, such that $\chi=1$ on a neighborhood of $\bar{V}$. There exists a $\mathbf{C}^{\infty}$ strictly plurisubharmonic exhaustion function $\phi$ on $\mathbb{C}^{n}$ such that $\phi<0$ on the set $K$ but $\phi>0$ on $\mathbb{C}^{n} \backslash V[4$, p. 110]. Choose a $\mathbf{C}^{\infty}$ function $h: \mathbb{R} \rightarrow[0, \infty)$ that is equal to 0 on $(-\infty, 0]$ and is strictly convex on $(0, \infty)$. Then the function

$$
\rho^{\prime}=\chi \circ \rho+c h \circ \phi: \mathbb{C}^{n} \rightarrow[0, \infty)
$$

is a strictly plurisubharmonic exhaustion function of class $\mathbf{C}^{2}$ on $\mathbb{C}^{n}$ provided that the constant $c>0$ is chosen sufficiently big, and $K=\left\{z \in \mathbb{C}^{n} \mid \rho^{\prime}(z)=0\right\}$. 
If $\psi \in E$ is small, the function $\tau=\rho^{\prime} \circ \Psi^{-1}$ is a small $\mathbf{C}^{2}$ perturbation of $\rho^{\prime}$, and $\tau=\rho^{\prime}$ outside a large compact subset $B$ of $\mathbb{C}^{n}$. Hence the Levi form $L_{\tau}=$ $\sum\left(\partial^{2} \rho / \partial z_{j} \partial \bar{z}_{k}\right) d z_{j} \otimes d \bar{z}_{k}$ of $\rho$ is a small perturbation of the Levi form $L_{\rho^{\prime}}$ of $\rho^{\prime}$, and they agree outside $B$. Since the eigenvalues of $L_{\rho^{\prime}}$ are positive on the compact set $B$, the same is true for $L_{\tau}$. This says that $\tau$ is a nonnegative strictly plurisubharmonic exhaustion function on $\mathbb{C}^{n}$. The approximation theorem [4, p. 119, Theorem 5.2.8] implies that the zero set of $\tau$ is polynomially convex. Since $\Psi(K)=\left\{z \in \mathbb{C}^{n} \mid \tau(z)\right.$ $=0\}$, Theorem 1.1 is proved.

Proof of Corollary 1.2. We may take $M=\mathbb{C}^{n}$ as before. Choose an open relatively compact neighborhood $V$ of $K$ in $N$. For each $t \in \mathbb{R}^{m}$ close to 0 the set $V_{t}=F_{t}(V)$ is a totally real submanifold of $\mathbb{C}^{n}$, and the map $\Phi_{t}: V_{0} \rightarrow V_{t}, \Phi_{t}=$ $F_{t} \circ F_{0}^{-1}$, is close to the identity map on $V_{0}$ in the $C^{2}$-sense. Since $\Phi_{t}\left(F_{0}(K)\right)=F_{t}(K)$, it suffices to show that there is an open neighborhood $U$ of $F_{0}(K)$ such that for each $t$ the map $\Phi_{t}$ can be extended to a map $\Psi^{t}$ on $U$ that is close to the identity in the $\mathrm{C}^{2}$-sense on $U$.

The map $\phi_{t}(z)=\Phi_{t}(z)-z, z \in V_{0}$, is small in the $C^{2}$-sense. Using a smooth partition of unity we extend $\phi_{t}$ to a $\mathbf{C}^{2}$ map $\psi_{t}$ on $U$ such that

$$
\left\|\psi_{t}\right\|_{\mathbf{C}^{2}(U)} \leqslant c\left\|\phi_{t}\right\|_{\mathbf{C}^{2}\left(V_{0}\right)}
$$

where the constant $c$ is independent of $t$. The map $\Psi_{t}(z)=z+\psi_{t}, z \in U$, is the desired extension of $\Phi_{t}$. Corollary 1.2 now follows from Theorem 1.1.

3. Perturbations on strictly pseudoconvex boundary points. We shall first consider the perturbations of $D$ that are supported in small subsets of $V$. Fix a point $x_{0} \in \Gamma \cap V$, an open neighborhood $V_{0}$ of $x_{0}$ such that $\bar{V}_{0} \subset V$, and a strictly plurisubharmonic defining function $\rho$ for $D \cap V_{0}$. According to [1, p. 530, Proposition 1] there exist a bounded strictly convex open set $C \subset \mathbb{C}^{n}(n=\operatorname{dim} M)$ with $\mathbf{C}^{2}$ boundary, a holomorphic map $\Phi: M \rightarrow \mathbb{C}^{n}$ and an open set $U \subset M, x_{0} \in U \subset \subset V_{0}$, such that the following hold:

(i) $\Phi(D) \subset C$,

(ii) $\Phi(\{z \in U \mid \rho(z)>0\}) \subset \mathbb{C}^{n} \backslash \bar{C}$,

(iii) $\Phi^{-1}(\Phi(U))=U$, and

(iv) the restriction $\left.\Phi\right|_{U}$ is regular and one-to-one.

Let $W$ be a neighborhood of $x_{0}$ such that $\bar{W} \subset U$. If $\Psi$ is a small $\mathbf{C}^{2}$ perturbation of $D$ supported in $W$, then $\tilde{\Psi}=\left.\Phi \circ \Psi \circ \Phi^{-1}\right|_{\Phi(U)}$ is a small $\mathbf{C}^{2}$ perturbation of $C$ supported in $\Phi(U)$. We choose $\Psi$ so close to the identity map that the set $\tilde{\Psi}(D)$ is still convex. For every point $x \in U \backslash \overline{\Psi(D)}$ we have $\Phi(x) \in \mathbb{C}^{n} \backslash \tilde{\Psi}(C)$ and hence there is a holomorphic function $h$ on $\mathbb{C}^{n}$ such that $h(\Phi(x))=1$, but $|h|<\frac{1}{2}$ on $\tilde{\Psi}(C)$. Because of (i) and (iii) above it follows that the point $x$ does not lie in the $\mathcal{O}(M)$-hull of $\overline{\Psi(D)}$.

To simplify the notation we write $K=\bar{D}$ and $K^{\prime}=\overline{\Psi(D)}$. The conclusion we just made is that

$$
\hat{K}^{\prime} \cap U=K^{\prime} \cap U
$$


where $\hat{K}^{\prime}$ is the $\mathcal{O}(M)$-convex hull of $K^{\prime}$. Since the support of $\Psi$ is contained in $W$, we have $K^{\prime} \backslash W=K \backslash W$, and hence (3.1) implies

$$
\hat{K}^{\prime} \cap(U \backslash W)=K \cap(U \backslash W) .
$$

We shall prove that $\hat{K}^{\prime}=K^{\prime}$. Assume that $\hat{K}^{\prime} \neq K^{\prime}$ in order to reach a contradiction. Because of (3.1) the two sets can differ only outside $U$. Since $K \backslash U=K^{\prime} \backslash U$, the set $\hat{K}^{\prime} \backslash U$ is strictly larger than $K \backslash U$. The polynomially convex set $K$ has a basis of open neighborhoods $\Omega$ that are smothly bounded strictly pseudoconvex domains with $\mathcal{O}(M)$-convex closure $\bar{\Omega}$. Thus we may choose $\Omega$ with these properties that does not contain the set $\hat{K}^{\prime} \backslash U$. By an embedding theorem for strictly pseudoconvex domains due to Fornaess [1, p. 543] and Khenkin [5, p. 668] there exists a holomorphic embedding $F: M \rightarrow \mathbb{C}^{N}$ for some $N \in \mathbb{Z}_{+}$and a bounded strictly convex domain $B \subset \mathbb{C}^{N}$ such that $F(\Omega) \subset B$ and $F(M \backslash \bar{\Omega}) \subset$ $\mathbb{C}^{N} \backslash \bar{B}$. We may assume that $0 \in B$. Let

$$
t_{0}=\inf \left\{t \in \mathbb{R}_{+} \mid F\left(\hat{K}^{\prime} \backslash U\right) \subset t \bar{B}\right\}
$$

and replace $B$ by $t_{0} B$. Then

$$
F\left(\hat{K}^{\prime} \backslash U\right) \subset \bar{B}
$$

and there is a point $p \in F\left(\hat{K}^{\prime} \backslash U\right) \cap b B$. The set $A=\mathbb{C}^{N} \backslash \overline{F(W)}$ is open and contains the point $p$. Moreover, it follows from (3.3) and (3.4) that $F\left(\hat{K}^{\prime}\right) \cap A \subset \bar{B}$. This means that locally near $p$ the polynomially convex set $F\left(\hat{K}^{\prime}\right)=\overline{F\left(K^{\prime}\right)}$ lies on the convex side of the smooth strictly convex hypersurface $b B$. According to $[\mathbf{1}, \mathrm{p}$. 530] there exists a holomorphic function $g$ defined on a neighborhood of $F\left(\hat{K}^{\prime}\right)$ in $\mathbb{C}^{N}$ such that

$$
g(p)=1 \text { and }|g(q)|<1 \quad \text { for } q \in F\left(\hat{K}^{\prime}\right) \backslash\{p\}
$$

If $\varepsilon>0$ is sufficiently small, the set

$$
F\left(\hat{K}^{\prime}\right) \cap\{|g| \leqslant 1-\varepsilon\} \varsubsetneqq F\left(\hat{K}^{\prime}\right)
$$

is polynomially convex and contains $F\left(K^{\prime}\right)$. This is a contradiction since $F\left(\hat{K}^{\prime}\right)$ $=\widehat{F\left(K^{\prime}\right)}$ is the polynomially convex hull of $F\left(K^{\prime}\right)$. This concludes the proof in the case when the support of the perturbation $\Psi$ is sufficiently small.

It remains to consider the general case. Let $\Gamma^{\prime}$ be an open relatively compact subset of $\Gamma \cap V$. Using the methods introduced by Fornaess in [1] we can show that there exist an open set $U \subset \subset V$ such that $U \cap \Gamma=\Gamma^{\prime}$, a holomorphic map $F$ : $M \rightarrow \mathbb{C}^{N}$ and a bounded strictly convex domain $C \subset \mathbb{C}^{N}$ with $\mathbf{C}^{2}$ boundary such that the properties (i)-(iv) above hold. Moreover, the map $F$ is transversal to $b C$ at every point $x \in \Gamma^{\prime}$. It follows that every small perturbation of $D$ supported in $U$ can be effected by a small perturbation of $C$ supported in a neighborhood of $F\left(\Gamma^{\prime}\right)$. The proof can be completed in the same way as above. We omit the details. 


\section{REFERENCES}

1. J. E. Fornaess, Embedding strictly pseudoconvex domains in convex domains, Amer. J. Math. 98 (1976), 529-569.

2. F. R. Harvey and R. O. Wells, Holomorphic approximation and hyperfunction theory on a $\mathbf{C}^{1}$ totally real submanifold of a complex manifold, Math. Ann. 197 (1972), 287-318.

3. Zero sets of non-negative strictly plurisubharmonic functions, Math. Ann. 201 (1973), $165-170$.

4. L. Hörmander, An introduction to complex analysis in several variables, North-Holland, Amsterdam and London, 1973.

5. G. M. Khenkin and E. M. Čirka, Boundary properties of holomorphic functions of several complex variables, Sovremeni Problemi Mat. 4, Moskva 1975, pp. 13-142; English transl., Soviet Math. J. 5 (1976), no. 5, 612-687. (Russian)

Department of Mathematics, University of Washington, Seattle, Washington 98195

Current address: Univerza E. K. V Ljubljani, FNT-Matematika, Jadranska 19, 61000 Ljubljana, Yugoslavia 